Groups Geom. Dyn. 8 (2014), 199-223

DOI $10.4171 / \mathrm{GGD} / 222$
Groups, Geometry, and Dynamics

(C) European Mathematical Society

\title{
Semigroups arising from asynchronous automata
}

\author{
David McCune
}

\begin{abstract}
In this paper we study semigroups of transformations of free monoids (and transformations of the corresponding tree boundaries) that arise from asynchronous automata. We introduce a subclass of asynchronous automata that we call "expanding automata". We show that every free partially commutative monoid is a synchronous automaton semigroup, and every free partially commutative semigroup is an expanding automaton semigroup. We show that undecidability arises in the actions of these semigroups on trees. In particular, in the class of asynchronous automata there is no algorithm which detects the presence of coincidence points and there is no algorithm which detects the presence of fixed points. We show that the classes of semigroups that arise from synchronous, expanding, and asynchronous automata are distinct classes of semigroups. We end the paper by covering some basic algebraic theory of these semigroups, with an emphasis on subgroups.
\end{abstract}

Mathematics Subject Classification (2010). 20M20; 20M35, $20 \mathrm{~B} 27$.

Keywords. Automaton, asynchronous, semigroup.

\section{Introduction}

Automaton groups (groups of automorphisms of labelled rooted trees generated by automata) have received systematic study since the 1980's. In particular, this class has been shown to contain many examples of groups with fascinating properties. For example, in 1983 Grigorchuk demonstrated an infinite finitely generated torsion group that was the first known group of intermediate growth (see [3]). Indeed, almost all known groups of intermediate growth are automaton groups. For an introduction to automaton groups, see Nekrashevych's book [11] or the survey paper [5] by Grigorchuk and S̆unić.

The automata used to generate automaton groups are invertible synchronous automata. Recently, many generalizations of automaton groups have been studied, all of these generalizations arising from generalizations of invertible synchronous automata. If we allow invertible synchronous automata to have infinitely many states, then groups generated by such automata are called self-similar. An introduction to these groups can be found in Nekrashevych's book [11]. In [15], Slupik and Sushchansky study semigroups arising from partial invertible synchronous au- 
tomata. Cain, Reznikov, Sushchansky, Silva, and Steinberg investigate automaton semigroups, which are semigroups that arise from (not necessarily invertible) synchronous automata (see [1], [12], and [14]). In [4], Grigorchuk et al. study groups arising from asynchronous automata. In this paper we investigate semigroups arising from asynchronous automata, and thus this paper contributes to the study of semigroups generated by automata.

In Section 2 we give all relevant definitions. We define a new subclass of asynchronous automata which we call "expanding automata" (an expanding automaton is an asynchronous automaton in which the range of the output function is the free semigroup generated by the alphabet, not the free monoid). We then define the semigroups associated with the different types of automata. Briefly, if $\mathcal{P}$ is a type of automaton (e.g. synchronous or expanding), then a $\mathcal{P}$ automaton semigroup is a semigroup that arises from a $\mathcal{P}$ automaton and which acts as transformations of a free monoid. Similarly, a $\partial-\mathcal{P}$ automaton semigroup is a semigroup that arises from a $\mathcal{P}$ automaton and which acts as partial transformations of the boundary of the regular rooted tree that is associated with a free monoid. We finish Section 2 by showing that each free partially commutative monoid is a synchronous automaton semigroup (Theorem 2.5), and refer the reader to [10] by the author for a proof that each free partially commutative semigroup is an expanding automaton semigroup.

In Section 3 we investigate the dynamics of the actions of these semigroups on regular rooted trees. In Lemma 3.1, we show that there is no algorithm that takes as input as expanding automaton $\mathcal{A}$ and two states $q$ and $r$ of $\mathcal{A}$ and decides whether or not $q$ and $r$ have a coincidence point in the corresponding tree (if $f, g: X \rightarrow Y$, then a coincidence point is a point $x$ such that $f(x)=g(x)$ ). Lemma 3.1 also shows the analogous result for the action on the boundary of the tree. We also show in Section 3 that there is no algorithm which takes as input an asynchronous automaton $\mathcal{A}$ and a state $q$ of $\mathcal{A}$ and decides whether or not $q$ has a fixed point in the corresponding tree. For this theorem, we again prove the analogous result for the action on the boundary of the tree. These last two results can be found in Theorem 3.3. We conclude Section 3 by demonstrating an algorithm that will find any fixed points of a transformation of the boundary of a tree where the transformation arises from an expanding automaton (Proposition 3.4).

In Section 4 we turn to describing the algebraic theory of these semigroups. We begin by showing that all of the various classes of semigroups are distinct classes. In particular, in Theorem 4.1 we show that synchronous automaton semigroups, expanding automaton semigroups, $\partial$-expanding automaton semigroups, asynchronous automaton semigroups, and $\partial$-asynchronous automaton semigroups are each a distinct class of semigroups. This theorem is proved through six propositions (Propositions 4.2-4.7). In Proposition 4.2 we give an infinite class of expanding automaton semigroups that are not synchronous automaton semigroups. In Proposition 4.3 we use residual finiteness to show that the class of expanding automaton semigroups is strictly contained in the class of asynchronous automaton semigroups. In Proposition 4.5 we show that the class of expanding automaton semigroups is strictly 
contained in the class of $\partial$-expanding automaton semigroups. In Proposition 4.6 we use the bicyclic monoid (the monoid with presentation $\operatorname{Mon}\langle a, b \mid a b=1\rangle$ ) to prove that the class of $\partial$-expanding automaton semigroups is strictly contained in the class of $\partial$-asynchronous automaton semigroups. We conclude section 4 by showing that the class of asynchronous automaton semigroups is strictly contained in the class of $\partial$-asynchronous automaton semigroups (Proposition 4.7).

In Section 5 we continue to study the basic algebraic theory of these semigroups, with an emphasis on subgroups. In Proposition 5.1 we show that expanding automaton semigroups are residually finite, and in Proposition 5.2 we show that the bicyclic monoid is not a submonoid of any $\partial$-expanding automaton semigroup. In Proposition 5.4 we show that a group $G$ is a $\partial$-expanding automaton semigroup if and only $G$ is an automaton group. In Proposition 5.5 we show that if $S$ is a $\partial$-expanding automaton semigroup with a unique maximal subgroup $G$, then $G$ is a self-similar group. This Proposition has as a corollary (Corollary 5.6) that if $\mathcal{A}$ is an invertible synchronous automaton, then the group of units of the semigroup generated by $\mathcal{A}$ is a self-similar group. We conclude the paper by showing that if $S$ is an asynchronous automaton semigroup and $G$ is a subgroup of $S$, then $G$ is residually finite (Proposition 5.7).

\section{Definitions and examples}

In this section we state the various definitions and notations required. We also give our first class of examples-we show that every partially commutative monoid and every partially commutative semigroup arise as asynchronous automaton semigroups. In particular, we give synchronous automata generating each free partially commutative monoid. We then refer the reader to Theorem 3.4.11 of [10] by the author for asynchronous automata giving each free partially commutative semigroup.

2.1. Automata and semigroups. We now define four different types of automata that we will use in this paper and the following. We first note that our definition of "automaton" differs from the definition found in the classical literature on automata. Hopcroft and Ullman in their book [7] refer to the automata in this paper as a specific kind of "transducer". We use "automaton" rather than "transducer" in order to agree with the literature on automaton groups.

Given a set $\Sigma$, let $\Sigma^{+}$denote the free semigroup generated by $\Sigma$, let $\Sigma^{*}$ denote the free monoid generated by $\Sigma$, and let $\Sigma^{\omega}$ denote the set of right-infinite words over $\Sigma$. We will always denote the identity element of a free monoid by the empty word $\emptyset$. Given a word $w \in \Sigma^{*}$ and a natural number $n \in \mathbb{N}$, let $w^{n}$ denote the word $w \ldots w$, where $w$ is written $n$ times.

To avoid ambiguity when presenting semigroups and monoids, we will use $S g\langle X \mid R\rangle$ to denote a semigroup presentation and $\operatorname{Mon}\langle X \mid R\rangle$ to denote a monoid presentation. 
Definition 2.1. An asynchronous automaton is a quadruple $\mathcal{A}=(Q, \Sigma, t, o)$ where $Q$ is a finite set of states, $\Sigma$ is a finite alphabet, $t: Q \times \Sigma \rightarrow Q$ is a transition function, and $o: Q \times \Sigma \rightarrow \Sigma^{*}$ is an output function.

We view an asynchronous automaton $\mathcal{A}=(Q, \Sigma, t, o)$ as a directed labelled graph with vertex set $Q$ and an edge from $q_{1}$ to $q_{2}$ labelled by $\sigma \mid w$ if and only if $t\left(q_{1}, \sigma\right)=q_{2}$ and $o\left(q_{1}, \sigma\right)=w$. If an edge of an automaton is labelled by $\sigma \mid w$, we call $\sigma$ the input letter of the edge and we call $w$ the output word of the edge.

Each state $q \in Q$ induces a function $f_{q}: \Sigma^{*} \rightarrow \Sigma^{*}$ in the following way. Let $w \in \Sigma^{*}$, and write $w=\sigma_{1} \ldots \sigma_{n}$. Then $f_{q}(w)$ is the word obtained by feeding $\sigma_{1} \ldots \sigma_{n}$ into the automaton as an input path (i.e., a path in the graph over the input letters) starting at $q$ and recording the corresponding outputs. By abuse of notation, we identify $f_{q}$ with $q$ as context should eliminate confusion.

Similarly, each state $q \in Q$ induces a (possibly) partial function $f_{q}^{\omega}: \Sigma^{\omega} \rightarrow \Sigma^{\omega}$. Given a right-infinite word $\eta \in \Sigma^{\omega}, f_{q}^{\omega}(\eta)$ is computed as in the previous paragraph: we view $\eta$ as an infinite path in the automaton starting at $q$ and $f_{q}^{\omega}(\eta)$ is defined to be the corresponding output path. We write $q^{\omega}$ for $f_{q}^{\omega}$, and we let $Q^{\Omega}$ denote the set $\left\{q^{\omega} \mid q \in Q\right\}$.

Example 2.2. Consider the automaton $\mathcal{A}=(Q, \Sigma, t, o)$ defined by

$$
\begin{gathered}
Q=\{a, b\}, \quad \Sigma=\{0,1\}, \\
t(a, 0)=a, \quad t(a, 1)=b, \quad t(b, 0)=t(b, 1)=b, \\
o(a, 0)=00, \quad o(a, 1)=1, \quad o(b, 0)=\emptyset, \quad o(b, 1)=1 .
\end{gathered}
$$

See Figure 1 for the graphical representation of $\mathcal{A}$. To compute $a(0010)$, for example, we view the word 0010 as a path in the automaton starting at $a$ over the input letters. The corresponding output path is $00001 \emptyset=00001$, and hence $a(0010)=$ 00001. Similarly, $b(0010)=\emptyset \emptyset 1 \emptyset=1$.

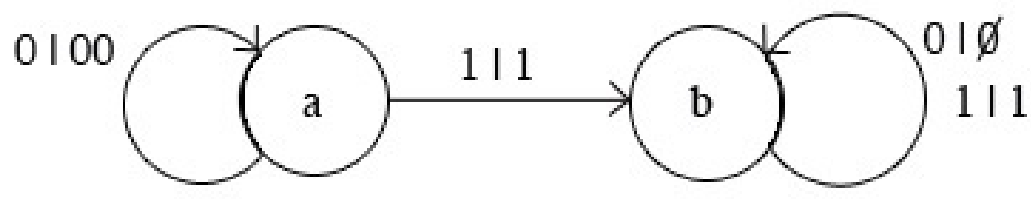

Figure 1. The automaton from Example 2.2.

To consider the action of $a^{\omega}$ and $b^{\omega}$ on $\Sigma^{\omega}$, note that $a^{\omega}(111 \cdots)=111 \cdots=$ $b^{\omega}(111 \cdots)$. On the other hand, $a^{\omega}(000 \cdots)=000 \cdots$ while $b^{\omega}(000 \cdots)=$ $\emptyset \emptyset \cdots=\emptyset$, and so $b^{\omega}(000 \cdots)$ is undefined. 
The set $\Sigma^{*}$ can be identified with a regular rooted tree of degree $|\Sigma|$, where the vertices of the tree are labelled by elements of $\Sigma^{*}$. The root vertex is labelled by $\emptyset$ and a vertex labelled $w$ has $|\Sigma|$ children whose labels are $w \sigma$ for each $\sigma \in \Sigma$. We do not distinguish between a vertex and its label unless context calls for a distinction. The action of a state $q$ on $\Sigma^{*}$ can thus be visualized as a transformation of the corresponding tree, where $q$ sends the vertex $w$ to the vertex $q(w)$. Note that $q$ induces a prefix-preserving transformation of $\Sigma^{*}$, i.e., if $v$ is a prefix of $w$ in $\Sigma^{*}$ then $q(v)$ is a prefix of $q(w)$. Furthermore, $q(\emptyset)=\emptyset$.

The set $\Sigma^{\omega}$ can be identified with the boundary of the tree corresponding to $\Sigma^{*}$. Let $k=|\Sigma|$. Then $\Sigma^{\omega}$ is a metric space with the distance between two rays $\eta$ and $\gamma$ defined by $d(\eta, \gamma)=k^{-|\eta \wedge \gamma|}$, where $|\eta \wedge \gamma|$ is the length of the longest common prefix of $\eta$ and $\gamma$. The space $\Sigma^{\omega}$ with this metric is homeomorphic to the Cantor set (its topology is the Tychonov product topology on $\Sigma^{\mathbb{N}}$, where $\Sigma$ has the discrete topology). Hence a transformation $q^{\omega}$ can be viewed as a continuous partial transformation of the boundary of a tree. For more details, see Section 2 of [4].

Let $\mathcal{A}=(Q, \Sigma, t, o)$ be an asynchronous automaton. There is a natural homomorphism $\phi_{\mathcal{A}}: Q^{+} \rightarrow \mathrm{p}-\mathrm{p} T\left(\Sigma^{*}\right)$, where $\mathrm{p}-\mathrm{p} T\left(\Sigma^{*}\right)$ denotes the semigroup of prefix-preserving transformations of $\Sigma^{*}$ that fix $\emptyset$. Similarly, there is a natural homomorphism $\psi_{\mathcal{A}}:\left(Q^{\Omega}\right)^{+} \rightarrow$ End $\partial \Sigma^{*}$, where End $\partial \Sigma^{*}$ denotes the semigroup of continuous partial transformations of the boundary of the tree. This leads to the following definition.

Definition 2.3. Let $\mathcal{A}=(Q, \Sigma, t, o)$ be an asynchronous automaton. Then the asynchronous automaton semigroup corresponding to $\mathcal{A}$, denoted $S(\mathcal{A})$, is the image of $\phi_{\mathcal{A}}$. The $\partial$-asynchronous automaton semigroup corresponding to $\mathcal{A}$, denoted $\partial S(\mathcal{A})$, is the image of $\psi_{\mathcal{A}}$. A semigroup $S$ is an asynchronous automaton semigroup if there exists an asynchronous automaton $\mathcal{A}$ with $S \cong S(\mathcal{A})$. Similarly, a semigroup $S$ is a $\partial$-asynchronous automaton semigroup if there exists an asynchronous automaton $\mathcal{A}$ with $S \cong \partial S(\mathcal{A})$.

Example. Let $\mathcal{A}$ be the automaton from Example 2.2. We compute $S(\mathcal{A})$ and $\partial S(\mathcal{A})$. To compute $S(\mathcal{A})$, first note that the range of $b$ is $\{1\}^{*}$ and $b$ fixes this set. Thus $b$ is idempotent. Since $a$ also fixes $\{1\}^{*}$, we have $a b=b$. Let $w \in\{0,1\}^{*}$, and write $w=0^{m_{1}} 1^{n_{1}} \ldots 0^{m_{k}} 1^{n_{k}}$. Let $r=\sum_{i=1}^{k} n_{i}$. Then

$$
b a(w)=b\left(0^{2^{m_{1}}} 1^{r}\right)=1^{r}=b(w),
$$

and so $b a=b$. Finally, note that $a$ is not periodic as $a^{m}(0)=0^{2^{m}}$. Thus $S(\mathcal{A})$ is the free semigroup of rank 1 with a zero adjoined, i.e.,

$$
S(\mathcal{A}) \cong S g\left\langle a, b \mid b^{2}=b, a b=b a=b\right\rangle .
$$

To compute $\partial S(\mathcal{A})$, note that the range of $b^{\omega}$ in $\{0,1\}^{\omega}$ is $111 \cdots$ and $b^{\omega}$ fixes $111 \cdots$. Hence $b^{\omega}$ is idempotent. Since $a^{\omega}$ fixes $111 \cdots, b^{\omega}$ is a zero element in 
the semigroup. Note that $a^{\omega}$ is not periodic as $\left(a^{\omega}\right)^{m}(0111 \cdots)=0^{2^{m}} 1 \cdots$. Hence $\partial S(\mathcal{A}) \cong S(\mathcal{A})$.

In the previous example, $\partial S(\mathcal{A}) \cong S(\mathcal{A})$. In general, this is not the case. To give a quick example, let $\mathcal{B}=(\{a\},\{0\}, t, o)$ where $t(a, 0)=a$ and $o(a, 0)=00$. Then $S(\mathscr{B})$ is the free semigroup of rank 1 but $\partial S(\mathscr{B})$ is the trivial semigroup. However, it is straightforward to show that for any automaton $\mathcal{A}, \partial S(\mathcal{A})$ is a quotient of $S(\mathcal{A})$.

We now give the remaining necessary definitions. An expanding automaton is an asynchronous automaton in which the range of the output function is $\Sigma^{+}$(rather than $\left.\Sigma^{*}\right)$. In the graphical representation of an expanding automaton, $\emptyset$ is never an output word. A synchronous automaton is an asynchronous automaton in which the range of the output function is $\Sigma$. In the graphical representation of a synchronous automaton, the output words are words of length one (i.e., the output words are output letters). An invertible synchronous automaton is a synchronous automaton in which, for each state $q$, the restricted function $\left.o\right|_{q \times \Sigma}: q \times \Sigma \rightarrow \Sigma$ is a permutation of $\Sigma$. In the graphical representation of an invertible synchronous automaton, for every state each letter of $\Sigma$ appears once and only once as an output letter.

Analogous to Definition 2.3, an expanding automaton semigroup is a semigroup $S$ such that there exists an expanding automaton $\mathcal{A}$ with $S(\mathcal{A}) \cong S$. A semigroup $S$ is a $\partial$-expanding automaton semigroup if there exists an expanding automaton $\mathcal{A}$ with $\partial S(\mathcal{A}) \cong S$. A synchronous automaton semigroup is defined similarly. It is known that if $\mathcal{A}$ is a synchronous automaton then $S(\mathcal{A}) \cong \partial S(\mathcal{A})$ (see Lemma 2.2 of [1]), so we do not discuss $\partial$-synchronous automaton semigroups. In the previous literature, synchronous automaton semigroups are simply referred to as "automaton semigroups"; we use the longer name to avoid ambiguity in the context of asynchronous automata. If $\mathcal{A}$ is an invertible automaton, then the states of the automaton induce graph automorphisms of the corresponding tree, and hence the states are invertible. Thus an automaton group is a group associated with an invertible synchronous automaton.

An automaton $\mathcal{A}=(Q, \Sigma, t, o)$ is infinite-state if $|Q|=|\mathbb{N}|$. If $\mathcal{P}$ is a type of automaton, then a semigroup $S$ is a $\mathcal{P}$ self-similar semigroup if $S \cong S(\mathcal{A})$ where $\mathcal{A}$ is a $\mathcal{P}$ automaton and $\mathcal{A}$ is allowed to be infinite-state. A $\partial-\mathcal{P}$ self-similar semigroup is defined analogously.

Let $T_{d}$ denote the transformation semigroup on $d$ objects and let $\Sigma=\{1, \ldots, d\}$. We write an element $t \in T_{d}$ as $\left[k_{1}, \ldots, k_{d}\right]$ where $t(i)=k_{i}$. Let $f \in \mathrm{p}$-p $T\left(\Sigma^{*}\right)$. Then $f$ decomposes as

$$
f=\tau_{f}\left(f_{1}, \ldots, f_{d}\right)
$$

where $\tau_{f} \in T_{d}$ and, for each $i \in \Sigma, f_{i} \in \mathrm{p}$-p $T\left(\Sigma^{*}\right)$ is characterized by the equation $f(i w)=\tau_{f}(i) f_{i}(w)$ for all $w \in \Sigma^{*}$. The function $f_{i}$ is called the section of $f$ at $i$. For any $v \in \Sigma^{*}$, we inductively define the section of $f$ at $v$ as the function $f_{v}$ where $f_{v}$ is characterized by the equation $f(v w)=f(v) f_{v}(w)$ for all $w \in \Sigma^{*}$. In terms of an automaton $\mathcal{A}=(Q, \Sigma, t, o)$, if $q \in Q$ then $q_{\sigma}=t(q, \sigma)$. Similarly, for 
any $v \in \Sigma^{+}, q_{v}$ is the state of $\mathcal{A}$ such that there is a path from $q$ to $q_{v}$ where the label of the path over the input letters is $v$. Thus when presenting automata we may just give the decompositions of the states as in Equation 1, as this is a quicker way of presenting an automaton than writing out the transition and output functions. For example, consider the automaton from Example 2.2 (see Figure 1). Then $a_{0}=a$ and $a_{1}=b$, and so $t(a, 0)=a$ and $t(a, 1)=b$. Similarly $o(a, 0)=00, o(a, 1)=1$, $t(b, 0)=b$, and so on.

Let $f=\tau_{f}\left(f_{1}, \ldots, f_{d}\right)$ and $g=\tau_{g}\left(g_{1}, \ldots, g_{d}\right)$. Then

$$
f \circ g=\left[f\left(\tau_{g}(1)\right), \ldots, f\left(\tau_{g}(d)\right)\right]\left(f_{\tau_{g}(1)} g_{1}, \ldots, f_{\tau_{g}(d)} g_{d}\right) .
$$

Given an automaton $\mathcal{A}=(Q, \Sigma, t, o)$ and an element $s \in S(\mathcal{A})$ where $s \notin Q$, Equation 2 allows us to construct a new automaton $\mathscr{B}$ such that $s$ is a state of $\mathscr{B}$ and $S(\mathscr{B}) \cong S(\mathcal{A})$. Thus, in many of the proofs below we will assume without loss of generality that a given element of an asynchronous automaton semigroup is a state of the given automaton. Finally, note that $(f \circ g)^{\omega}=f^{\omega} \circ g^{\omega}$, and so we can make the same assumptions when considering $\partial$-asynchronous automaton semigroups.

2.2. Free partially commutative monoids. In this section, we give automata generating each partially commutative monoid. A free partially commutative monoid is a monoid with presentation $\operatorname{Mon}\left\langle x_{1}, \ldots, x_{n} \mid R\right\rangle$ where $R \subseteq\left\{\left(x_{i} x_{j}, x_{j} x_{i}\right) \mid 1 \leq\right.$ $i, j \leq n\}$. A free partially commutative semigroup is defined analogously.

Let $M$ be a free partially commutative monoid with presentation $\operatorname{Mon}\langle X \mid R\rangle$. We begin by defining the shortlex normal form on $M$. First, if $v \in X^{*},|v|$ will always denote the length of $v$ in $X^{*}$. Order the set $X$ by $x_{i}<x_{j}$ whenever $i<j$. If $v, w \in X^{*}$, let $v<w$ if and only if $|v|<|w|$ or, if $|v|=|w|, v$ comes before $w$ in the dictionary order induced by the order on $X$. This is called the shortlex ordering on $X^{*}$. To obtain the set of shortlex normal forms of $M$, for each $w \in M$ choose a word $w^{\prime} \in X^{*}$ such that $w=w^{\prime}$ in $M$ and $w^{\prime}$ is minimal in $X^{*}$ with respect to the shortlex ordering. We remark that it is immediate from this definition that a word $w \in X^{*}$ is in shortlex normal form in $M$ if and only if for all factorizations $x=y b u a z$ in $M$ where $y, u, z \in X^{*}, a$ and $b$ commute, and $a<b$, there is a letter of $u$ which does not commute with $a$.

For any $w \in X^{*}$, let $w\left(x_{i}, x_{j}\right)$ denote the word obtained from $w$ by erasing all letters except $x_{i}$ and $x_{j}$. We write $w\left(x_{i}\right)$ to denote the word obtained from $w$ by deleting all letters except $x_{i}$. We will need the following lemma regarding free partially commutative monoids.

Lemma 2.4. Let $M$ be a free partially commutative monoid generated by $X=$ $\left\{x_{1}, \ldots, x_{n}\right\}$, and let $v, w \in X^{*}$ such that $v$ and $w$ are in shortlex normal form in $M$. Suppose that

(1) $\left|v\left(x_{i}\right)\right|=\left|w\left(x_{i}\right)\right|$ for $1 \leq i \leq n$ and

(2) $v\left(x_{i}, x_{j}\right)=w\left(x_{i}, x_{j}\right)$ in $X^{*}$ whenever $1 \leq i, j \leq n$ and $x_{i}$ and $x_{j}$ do not commute. 
Then $v=w$ in $M$.

Proof. Let $v, w \in M$ be words satisfying $\left|v\left(x_{i}\right)\right|=\left|w\left(x_{i}\right)\right|$ for all $i$. This implies that the number of occurrences of $x_{i}$ as a letter of $v$ equals the number of occurrences of $x_{i}$ as a letter of $w$. In particular, $|v|=|w|$. Write $v=x_{i_{1}} \ldots x_{i_{k}}$ and $w=x_{j_{1}} \ldots x_{j_{k}}$ with $v, w$ in shortlex normal form. Suppose that $x_{i_{1}}<x_{j_{1}}$. Then $v\left(x_{i_{1}}, x_{j_{1}}\right) \neq w\left(x_{i_{1}}, x_{j_{1}}\right)$ in $X^{*}$, and condition (2) in the hypotheses implies that $x_{i_{1}}$ and $x_{j_{1}}$ commute. Condition (1) implies that $x_{j_{1}}$ is a letter of $v$ and $x_{i_{1}}$ is a letter of $w$, and so we write $v=x_{i_{1}} v_{1} x_{j_{1}} v_{2}$ where $v_{1}$ does not contain $x_{j_{1}}$ as a letter. Similarly, write $w=x_{j_{1}} w_{1} x_{i_{1}} w_{2}$. Condition (2) implies that $x_{i_{1}}$ commutes with every letter of $w_{1}$. Since $x_{i_{1}}<x_{j_{1}}$, we have that $w$ was not in lexicographic normal form. Thus $x_{i_{1}} \nless x_{j_{1}}$, and symmetry implies $x_{j_{1}} \nless x_{i_{1}}$. So $x_{i_{1}}=x_{j_{1}}$. Inductively continuing the argument implies that $x_{i_{t}}=x_{j_{t}}$ for all $1 \leq t \leq k$.

Theorem 2.5. Every free partially commutative monoid is a synchronous automaton semigroup.

Proof. Let $M$ be a partially commutative monoid generated by $X=\left\{x_{1}, \ldots, x_{n}\right\}$. Let $N=\left\{\{i, j\} \mid x_{i}\right.$ and $x_{j}$ do not commute $\}$. Let $A=\left\{a_{1}, \ldots, a_{n}\right\}, B=$ $\left\{b_{1}, \ldots, b_{n}\right\}, C=\left\{c_{i j} \mid i<j\right.$ and $\left.\{i, j\} \in N\right\}$, and $D=\left\{d_{i j} \mid i<j\right.$ and $\{i, j\} \in$ $N$ \} be four alphabets where $C, D$ are in bijective correspondence with $N$. We construct an automaton $\mathcal{A}_{M}$ with state set $Q:=\left\{y_{1}, \ldots, y_{n}, 1\right\}$ over the alphabet $\Sigma=A \cup B \cup C \cup D$ such that $S\left(\mathcal{A}_{M}\right) \cong M$ as follows. Let 1 be the sink state that pointwise fixes $\Sigma^{*}$. For each $i$, define

$$
t\left(y_{i}, a_{j}\right)=1 \text { for all } j, \quad t\left(y_{i}, b_{j}\right)= \begin{cases}y_{i}, & i=j, \\ 1, & i \neq j,\end{cases}
$$

and

$$
o\left(y_{i}, a_{j}\right)=\left\{\begin{array}{ll}
b_{j}, & i=j, \\
a_{j}, & i \neq j,
\end{array} \quad o\left(y_{i}, b_{j}\right)= \begin{cases}a_{j}, & i=j, \\
b_{j}, & i \neq j .\end{cases}\right.
$$

By construction, the subautomaton consisting of the states $y_{i}$ and 1 over the alphabet $\left\{a_{i}, b_{i}\right\}$ is the adding machine automaton (see Figure 1.3 of [11]) for all $i$. Note that for any $k>j, y_{i}^{j}\left(a_{i}^{2 j}\right) \neq y_{i}^{k}\left(a^{2 j}\right)$, and so the semigroup corresponding to this subautomaton is the free monoid of rank 1 for all $i$. Thus each $y_{i}$ acts nonperiodically on $\left\{a_{i}, b_{i}\right\}^{*}$ for all $i$. Furthermore, if $i \neq j$ then $y_{j}$ induces the identity function from $x \Sigma^{*}$ to $x \Sigma^{*}$ where $x \in\left\{a_{i}, b_{i}\right\}$.

We now complete the construction of $\mathcal{A}$. Fix $i<j$ with $\{i, j\} \in N$, and let $k \in \mathbb{N}$ such $1 \leq k \leq n$ and $k \neq i, j$. Define

$$
\begin{gathered}
t\left(y_{i}, c_{i j}\right)=y_{j}, \quad t\left(y_{i}, d_{i j}\right)=y_{i}, \quad t\left(y_{j}, c_{i j}\right)=y_{i}, \quad t\left(y_{j}, d_{i j}\right)=y_{j}, \\
o\left(y_{i}, c_{i j}\right)=d_{i j}, \quad o\left(y_{i}, d_{i j}\right)=c_{i j}, \quad o\left(y_{j}, c_{i j}\right)=c_{i j}, \quad o\left(y_{j}, d_{i j}\right)=d_{i j},
\end{gathered}
$$




$$
\begin{gathered}
t\left(y_{k}, c_{i j}\right)=t\left(y_{k}, d_{i j}\right)=1, \\
o\left(y_{k}, c_{i j}\right)=c_{i j}, \quad t\left(y_{k}, d_{i j}\right)=d_{i j} .
\end{gathered}
$$

For all other $i^{\prime}, j^{\prime}$ such that $\left\{i^{\prime}, j^{\prime}\right\} \subseteq N$ and $i^{\prime}<j^{\prime}$, define the output and transition function analogously. Figure 2 gives the automaton $\mathcal{A}_{M}$ where $M$ is the free partially commutative monoid Mon $\left\langle y_{1}, y_{2}, y_{3} \mid y_{1} y_{2}=y_{2} y_{1}, y_{1} y_{3}=y_{3} y_{1}\right\rangle$ (we omit the arrow on the identity state).

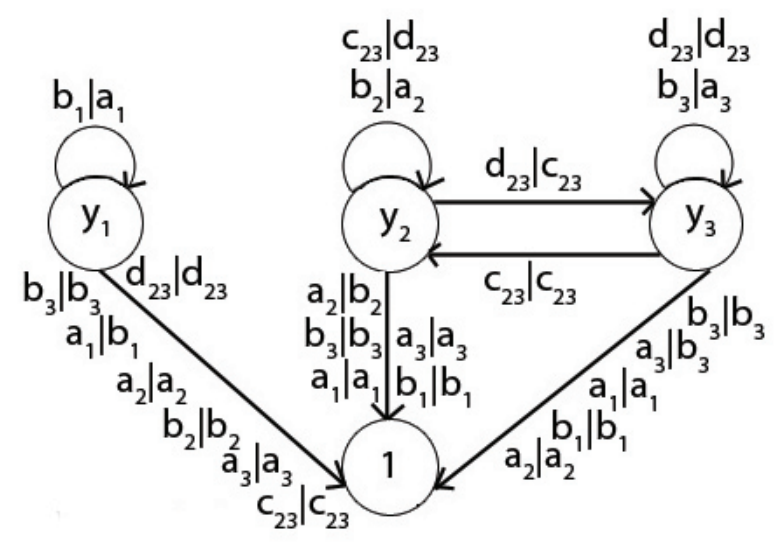

Figure 2. An automaton giving the monoid $\operatorname{Mon}\left\langle y_{1}, y_{2}, y_{3} \mid y_{1} y_{2}=y_{2} y_{1}, y_{1} y_{3}=y_{3} y_{1}\right\rangle$.

For each $\{i, j\} \in N$, the subautomaton of $\mathcal{A}_{M}$ corresponding to the states $y_{i}$ and $y_{j}$ over the alphabet $\left\{c_{i j}, d_{i j}\right\}$ is the "lamplighter automaton" (see Figure 1.1 of [6]). Grigorchuk and Zuk show in Theorem 2 of [6] that this automaton generates the lamplighter group, and in particular in Lemma 6 of [6] they show that the states of this automaton generate a free semigroup of rank 2. Thus $y_{i}$ and $y_{j}$ generate a free semigroup of rank 2 when acting on $\left\{c_{i j}, d_{i j}\right\}^{*}$, and hence the semigroup generated by $y_{i}$ and $y_{j}$ in $S\left(\mathcal{A}_{M}\right)$ is free of rank 2 .

Let $1 \leq i, j \leq n$ be such that $\{i, j\} \nsubseteq N$. By construction of $\mathcal{A}_{M}, y_{i}$ and $y_{j}$ have disjoint support, i.e., the sets $\left\{w \in \Sigma^{*} \mid y_{i}(w) \neq w\right\}$ and $\left\{w \in \Sigma^{*} \mid y_{j}(w) \neq w\right\}$ are disjoint. Thus if $x_{i}$ and $x_{j}$ commute in $M$, then $y_{i}$ and $y_{j}$ commute in $S\left(\mathcal{A}_{M}\right)$. So $S\left(\mathcal{A}_{M}\right)$ is a quotient of $M$.

Let $v, w \in Q^{*}$ such that $v$ and $w$ are written in shortlex normal form when considered as elements of $M$. Suppose that $w\left(y_{i}\right) \neq v\left(y_{i}\right)$ for some $i$. By construction of $\mathcal{A}_{M}$, for any $i \neq j$ we have $y_{j}$ acts as the identity function on $\left\{a_{i}, b_{i}\right\}^{*}$. Thus the action of $v$ and $w$ on $\left\{a_{i}, b_{i}\right\}^{*}$ is the same as the action of $v\left(y_{i}\right)$ and $w\left(y_{i}\right)$, respectively, on $\left\{a_{i}, b_{i}\right\}^{*}$. So $w\left(y_{i}\right) \neq v\left(y_{i}\right)$ implies that $v \neq w$ in $S\left(\mathscr{A}_{M}\right)$. Hence $v=w$ in $S\left(\mathcal{A}_{M}\right)$ implies that $w\left(y_{i}\right)=v\left(y_{i}\right)$ for all $i$.

Suppose now that there exist $\{r, s\} \in N$ such that $v\left(y_{r}, y_{s}\right) \neq w\left(y_{r}, y_{s}\right)$. If $t \neq r, s$, then $y_{t}$ acts like the identity function on $\left\{c_{r s}, d_{r s}\right\}^{*}$. Thus the action of $v$ and $w$ on $\left\{c_{r s}, d_{r s}\right\}^{*}$ is the same as the action of $v\left(y_{r}, y_{s}\right)$ and $w\left(y_{r}, y_{s}\right)$, respectively, 
on $\left\{c_{r s}, d_{r s}\right\}^{*}$. So $v\left(y_{r}, y_{s}\right) \neq w\left(y_{r}, y_{s}\right)$ implies that $v \neq w$ in $S\left(\mathcal{A}_{\mathcal{M}}\right)$. Thus if $v=w$ in $S\left(\mathcal{A}_{\mathcal{M}}\right)$ then $v\left(y_{r}, y_{s}\right)=w\left(y_{r}, y_{s}\right)$ in $Q^{*}$ for all $\{r, s\} \in N$.

The last two paragraphs have shown that if $v=w$ in $S\left(\mathcal{A}_{\mathcal{M}}\right)$, then $v$ and $w$ satisfy the hypotheses of Lemma 2.4. Hence $v=w$ in $M$, and the result follows.

It is shown in Proposition 4.3 of [1] that the free semigroup of rank 1 is not a synchronous automaton semigroup. The free semigroup of rank 1 is a free partially commutative semigroup, so not all free partially commutative semigroups are synchronous automaton semigroups. However, it is currently unknown if any other free partially commutative semigroups are not synchronous automaton semigroups. In particular, in [1] Cain provides synchronous automata giving each free semigroup of rank at least 2 and each free commutative semigroup of rank at least 2, so there are large classes of free partially commutative semigroups that arise in the class of synchronous automaton semigroups. In any case, the author provides expanding automata that give each free partially commutative semigroup in Theorem 3.4.11 of [10]. The proof scheme is essentially that of the proof of Theorem 2.5.

\section{Dynamics of asynchronous automaton semigroups}

The goal of this section is to show that there is no algorithm which takes as input an asynchronous automaton $\mathcal{A}$ over an alphabet $\Sigma$ and an element $s \in S(\mathcal{A})$ and decides whether or not $s$ has a fixed point in $\Sigma^{+}$. We show the analogous statement for $\partial-$ asynchronous automaton semigroups, i.e., we show that there is no algorithm which takes as input an asynchronous automaton $\mathcal{A}$ over an alphabet $\Sigma$ and an element $s \in \partial S(\mathcal{A})$ and decides whether or not $s$ has a fixed point in $\Sigma^{\omega}$. These results show that the actions of asynchronous automaton semigroups are more complicated than the actions of automaton groups, as there is an algorithm that takes as input an automaton group $G$ and an element $g \in G$ and decides whether or not $g$ has a fixed point in the corresponding tree. There is also an algorithm which decides whether or not $g_{\omega}$ has a fixed point in the boundary of the corresponding tree. These two algorithms for automaton groups are straightforward.

Let $f, g$ be maps $X \rightarrow Y$. A point $x \in X$ is a coincidence point of $f$ and $g$ if $f(x)=g(x)$. We begin this section by showing that there is no algorithm that decides if two elements of an expanding automaton semigroup have a coincidence point.

Before we show the results regarding coincidence points, we mention that Grigorchuk et al. show in Proposition 2.8 of [4] that the uniform word problem is decidable for $\partial$-asynchronous automaton semigroups. We show in Section 4 below that every asynchronous automaton semigroup is a $\partial$-asynchronous automaton semigroup, and hence the uniform word problem is decidable for the class of asynchronous automaton semigroups. Thus there is an algorithm that takes as input a $\partial$-asynchronous automaton semigroup $S$ over an alphabet $\Sigma$ and $s_{1}, s_{2} \in S$ and decides whether or not 
$s_{1}(\eta)=s_{2}(\eta)$ for all $\eta \in \Sigma^{\omega}$. We show below that there is no algorithm that takes as input an $\partial$-expanding automaton semigroup $S$ over an alphabet $\Sigma$ and $s_{1}, s_{2} \in S$ and decides if there exists an infinite word $\eta \in \Sigma^{\omega}$ such that $s_{1}(\eta)=s_{2}(\eta)$. Lemma 3.1 is therefore interesting in its own right, as it shows that a decision problem that is obtained from slightly tweaking the word problem turns out to be undecidable.

Lemma 3.1. There is no algorithm which takes as input an expanding automaton $\mathcal{A}=(Q, \Sigma, t, o)$ and states $q, r \in Q$ and decides whether or not $q$ and $r$ (respectively $q^{\omega}$ and $\left.r^{\omega}\right)$ have a coincidence point in $\Sigma^{+}$(respectively $\left.\Sigma^{\omega}\right)$.

Proof. We show undecidability by embedding the Post Correspondence Problem. Let $X=\left\{x_{1}, \ldots, x_{m}\right\}$ be an alphabet, and let $V=\left(v_{1}, \ldots, v_{n}\right)$ and $W=\left(w_{1}, \ldots, w_{n}\right)$ be two lists of words over $X$. Let $Y=\{1, \ldots, n\} \subseteq \mathbb{N}$ and $Z=\left\{z_{1}, z_{2}\right\}$ be alphabets such that $X \cap Y \cap Z=\emptyset$. Undecidability of the Post Correspondence Problem implies that, in general, we cannot decide if there is a sequence $\left(y_{1}, \ldots, y_{t}\right)$ of elements of $Y$ such that $v_{y_{1}} v_{y_{2}} \ldots v_{y_{t}}=u_{y_{1}} u_{y_{2}} \ldots u_{y_{t}}$.

We build an expanding automaton $\mathcal{A}_{X, V, W}$ over the alphabet $\Sigma:=X \cup Y \cup Z$ as follows. Let the state set $Q$ of $\mathcal{A}_{X, V, W}$ be $\{a, b\}$, and let

$$
\begin{gathered}
t(q, \sigma)=q \text { for all } q \in Q, \sigma \in \Sigma, \\
o(a, i)=v_{i} \text { for } 1 \leq i \leq n, \quad o(a, \sigma)=z_{1} \text { for } \sigma \in \Sigma-Y, \\
o(b, i)=w_{i} \text { for } 1 \leq i \leq n, \quad o(b, \sigma)=z_{2} \text { for } \sigma \in \Sigma-Y .
\end{gathered}
$$

Figure 3 shows $\mathcal{A}_{X, U, W}$ where $X=\{s, t\}, V=\left(s t, t s^{2}, t^{2}\right)$, and $W=\left(s^{2}, t s t s, t^{2} s\right)$.
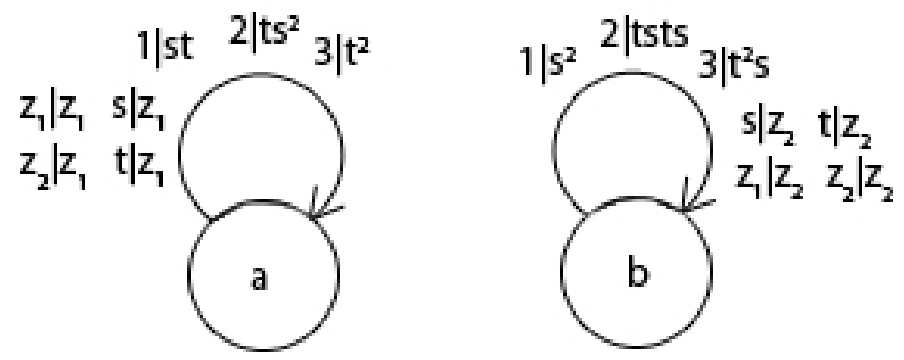

Figure 3. The automaton $\mathcal{A}_{X, U, W}$ where $X=\{s, t\}, V=\left(s t, t s^{2}, t^{2}\right)$, and $W=$ $\left(s^{2}, t s t s, t^{2} s\right)$.

Note that for any $w \in \Sigma^{*}, a(w)$ does not contain the letter $z_{2}$; similarly, $b(w)$ does not contain the letter $z_{1}$. Now if $w \in \Sigma^{*}$ contains a letter of $X \cup Z$, then we know $a(w) \neq b(w)$ since $a(w)$ contains the letter $z_{1}$ and $b(w)$ contains the letter $z_{2}$. Thus if there is a word $w \in \Sigma^{*}$ such that $a(w)=b(w)$, then $w \in Y^{*}$. 
By construction of $\mathcal{A}_{X, V, W}$, if $y=y_{1} y_{2} \ldots y_{n} \in Y^{*}$ and $a(y)=b(y)$, then $v_{y_{1}} v_{y_{2}} \ldots v_{y_{t}}=u_{y_{1}} u_{y_{2}} \ldots u_{y_{t}}$. Thus the expanding automaton $\mathcal{A}_{X, V, W}$ simulates Post's problem, and since we cannot decide the Post Correspondence Problem, we cannot decide if there is a word $w \in Y^{*}$ with $a(w)=b(w)$. This proves part (1).

It is shown by Rouhonen in [13] that the infinite Post Correspondence Problem is undecidable. That is, there is no algorithm that takes as input two lists of words $v_{1}, \ldots, v_{n}$ and $w_{1}, \ldots, w_{n}$ over an alphabet $X$ and decides if there is an infinite sequence $\left(i_{k}\right)_{k=1}^{\infty}$ such that $v_{i_{1}} v_{i_{2}} \cdots=w_{i_{1}} w_{i_{2}} \ldots$ Thus, using the same expanding automata and logic as above, the corresponding decision problem on the boundary is also undecidable.

We now show that undecidability arises when trying to understand the fixed point sets of elements of asynchronous automaton semigroups. If $w \in A^{*}$ for a set $A$, let $\operatorname{Pref}_{k}(w)$ denote the prefix of $w$ of length $k$.

Definition 3.2. Let $A^{*}$ be a free monoid. A subset $C \subseteq A^{*}$ is a prefix code if

(1) $C$ is the basis of a free submonoid of $A^{*}$,

(2) if $c \in C$, then $\operatorname{Pref}_{k}(c) \notin C$ for all $1 \leq k \leq|C|$.

The prefix code Post correspondence problem is a stronger form of the Post Correspondence Problem. The input of the prefix code Post Correspondence Problem is two lists of words $v_{1}, \ldots, v_{n}$ and $w_{1}, \ldots, w_{n}$ over an alphabet $X$ such that $\left\{v_{1}, \ldots, v_{n}\right\}$ and $\left\{w_{1}, \ldots, w_{n}\right\}$ are prefix codes. A solution to the problem is a sequence of indices $\left(i_{k}\right)_{1 \leq k \leq m}$ with $1 \leq i_{k} \leq n$ such that $v_{i_{1}} \ldots v_{i_{m}}=w_{i_{1}} \ldots w_{i_{m}}$. Rouhonen also shows in [13] that this form of Post's problem is undecidable. We use the prefix code Post problem to prove the following:

Theorem 3.3. There is no algorithm that takes as input an asynchronous automaton $\mathcal{A}$ over an alphabet $\Sigma$ and a state $q$ of $\mathcal{A}$ and decides whether or not $q$ (respectively $\left.q^{\omega}\right)$ has a fixed point in $\Sigma^{+}\left(\right.$respectively $\left.\Sigma^{\omega}\right)$.

Proof. Let $\Sigma$ be an alphabet and let $C, D \subseteq \Sigma^{*}$ be prefix codes where $C=$ $\left\{c_{1}, \ldots, c_{m}\right\}$ and $D=\left\{d_{1}, \ldots, d_{m}\right\}$. Let $\mathcal{A}_{\Sigma, C, D}$ be the expanding automaton with states $c, d$ that we constructed in the proof of Proposition 3.1. Then $\mathcal{A}_{\Sigma, C, D}$ is an expanding automaton over the alphabet $X:=\{1, \ldots, m\} \cup \Sigma \cup\left\{z_{1}, z_{2}\right\}$ such that $o(c, i)=c_{i}$ and $o(d, i)=d_{i}$. We build an asynchronous automaton $\mathscr{B}$ over the alphabet $X$ with a state $c^{\prime}$ such that $c^{\prime} c$ is the identity function from $\{1, \ldots, m\}^{*}$ to $\{1, \ldots, m\}^{*}$. We know that there is a function $c^{\prime}: \Sigma^{*} \rightarrow\{1, \ldots, m\}^{*}$ such that $c^{\prime} c$ is the identity because $\left\{c_{1}, \ldots, c_{m}\right\}$ generates a free monoid, so $c$ induces an injection from $\{1, \ldots, m\}^{*}$ to $\Sigma^{*}$.

We begin construction of $\mathscr{B}$ by starting with a single state $c^{\prime}$, and then attaching a loop based at $c^{\prime}$ such that the input letters of the loop read the word $c_{1}$ when read starting at $c^{\prime}$. We define the corresponding output word, when read starting at $c^{\prime}$, to be 
$(\emptyset)^{\left|c_{1}\right|-1} 1$. In other words, the first $\left|c_{1}\right|-1$ edges of the loop have the form $x \mid \emptyset$, and the last edge of the loop has the form $x \mid 1$. Next, we attach a loop at $c^{\prime}$ such that the input letters of the loop when read starting at $c^{\prime}$ read the word $c_{2}$, and the corresponding output word is $(\varnothing)^{\left|c_{2}\right|-1} 2$. If $c_{1}$ ad $c_{2}$ have a non-trivial common prefix, then the resulting automaton with two loops is not deterministic. In this case, we "fold" the maximum length common prefixes together, resulting in a deterministic automaton. We iteratively continue this process until we can read the words $c_{1}, \ldots, c_{m}$ as input words starting at $c^{\prime}$, and $c^{\prime}\left(c_{i}\right)=i$ for all $i$. Note that we can do this process since $c_{i}$ is not a prefix of $c_{j}$ for any $i \neq j$. At this step in the construction of $\mathcal{B}, \mathcal{B}$ is a partial asynchronous automaton, i.e., given a state of $q$ of $\mathcal{B}$, the domain of $q$ is not all of $X^{*}$. However, we do have $c^{\prime} c$ is the identity function $\{1, \ldots, m\}^{*} \rightarrow\{1, \ldots, m\}^{*}$ by construction of $\mathcal{B}$. In order to make $\mathcal{B}$ an asynchronous automaton, for each state $q$ in $\mathscr{B}$ and each letter $x \in X$ such that $t(q, x)$ is undefined, let $t(q, x)=q$ and $o(q, x)=\varnothing$.

Recall that in the proof of Lemma 3.1, in general we cannot find $w \in\{1, \ldots, m\}^{*}$ such that $c(w)=d(w)$ because such a $w$ is a solution to the Post Correspondence Problem. By construction of $\mathcal{B}$, any $w \in\{1, \ldots, m\}^{*}$ such that $c^{\prime} d(w)=w=$ $c^{\prime} c(w)$ is a solution to the prefix code Post Correspondence Problem. Now $c^{\prime} d$ is an element of the asynchronous automaton semigroup generated by the states of $\mathcal{A}_{\Sigma, C, D}$ and $\mathcal{B}$. Thus, undecidability of the prefix code Post Correspondence Problem implies part (1).

In [13], Ruohonen shows that the that there is no algorithm which takes as input two lists of words $v_{1}, \ldots, v_{n}$ and $w_{1}, \ldots, w_{n}$ over an alphabet $\Sigma$ such that $\left\{v_{1}, \ldots, v_{n}\right\}$ and $\left\{w_{1}, \ldots, w_{n}\right\}$ are prefix codes and decides whether there is an infinite sequence of indices $\left(i_{k}\right)_{k=1}^{\infty}$ such that $v_{i_{1}} v_{i_{2}} \ldots=w_{i_{1}} w_{i_{2}} \ldots$ Thus, using the same constructions and logic as above, the corresponding decision problem on the boundary is also undecidable.

We close this section by showing that there is an algorithm that decides whether or not an element of a $\partial$-expanding automaton semigroup has a fixed point in the boundary of the corresponding tree. To do so, we need the following definition: if $\mathcal{A}$ is an automaton, then an inactive path in $\mathcal{A}$ is a path in $\mathcal{A}$ such that each edge of the path has a label of the form $\sigma \mid \sigma$ (we also count the empty path as inactive). The logic of the following proof can mostly be found in Section 2.1 of Lothaire's book [9].

Proposition 3.4. There is an algorithm that takes as input an expanding automaton $\mathcal{A}=(Q, \Sigma, t, o)$ and a state $q \in Q$ and decides whether or not $q^{\omega}$ has a fixed point in $\Sigma^{\omega}$.

Proof. Suppose first that there is an inactive circuit in $\mathcal{A}$ that is accessible from $q$ via an inactive path. Then the infinite word obtained from traveling along the inactive path and then winding around the inactive circuit is a fixed point of $q^{\omega}$.

Suppose next that there is an edge coming from $q$ with a label of the form $\sigma \mid \sigma w$ where $w \in \Sigma^{+}$. Then a quick induction shows that $q^{n}(\sigma)$ is a prefix of $q^{n+1}(\sigma)$ for 
all $n \in \mathbb{N}$. Inductively, write $q^{n}(\sigma)=\sigma w w_{1} \ldots w_{n-1}$ where $q\left(\sigma w w_{1} \ldots w_{n-3}\right)=$ $\sigma w w_{1} \ldots w_{n-2}$. Let $\eta=0 w w_{1} w_{2} \ldots$, i.e., $\eta=\lim _{n \rightarrow \infty} q^{n}(\sigma)$. Note that $\eta$ is an infinite word because $\mathcal{A}$ is an expanding automaton. Then $\eta$ is a fixed point of $q^{\omega}$, and in fact $\eta$ is the only fixed point of $q^{\omega}$ that begins with the letter $\sigma$ (see Section 2.1 of [9], where Lothaire uses the same procedure to obtain the Thue-Morse sequence).

The previous paragraphs show that if (a) there is an inactive circuit accessible from $q$ via an inactive path or (b) there is a state $q^{\prime}$ with an edge of the form $\sigma \mid \sigma w$ where $w \in \Sigma^{+}$such that $q^{\prime}$ is accessible from $q$ via an inactive path, then $q^{\omega}$ has a fixed point in $\Sigma^{\omega}$. We show that the converse is also true.

Suppose that neither (a) nor (b) holds. Since (a) does not hold, there are finitely many inactive paths in $\mathcal{A}$ beginning at $q$. Let $q_{1}, \ldots, q_{m}$ be the terminal vertices of the inactive paths. Since condition (b) does not hold, for each $1 \leq i \leq m$ every edge beginning at $q_{i}$ has a label of the form $\sigma \mid \sigma^{\prime} w$, where $\sigma^{\prime} \in \Sigma-\{\sigma\}$ and $w \in \Sigma^{*}$. Thus $q^{\omega}$ does not have a fixed point, and we have shown the converse.

We conclude this section with the following remark, which reiterates one of the ways in which a state of an expanding automaton can have a fixed point on the boundary of the corresponding tree.

Remark 3.5. Let $\mathcal{A}=(Q, \Sigma, t, o)$ be an expanding automaton and let $q \in Q$. If there is an edge coming from $q$ with a label of the form $\sigma \mid \sigma w$ for some $w \in \Sigma^{+}$, then $q^{\omega}$ has exactly one fixed point in $\sigma \Sigma^{\omega}$.

\section{Distinguishing classes of semigroups}

The goal of this section is to distinguish the various classes of semigroups. In particular, we show the following theorem.

Theorem 4.1. Let $S A S$ denote the class of synchronous automaton semigroups, EAS denote the class of expanding automaton semigroups, $\partial E A S$ denote the class of $\partial$ expanding automaton semigroups, AAS denote the class of asynchronous automaton semigroups, and $\partial A A S$ denote the class of $\partial$-asynchronous automaton semigroups. Then

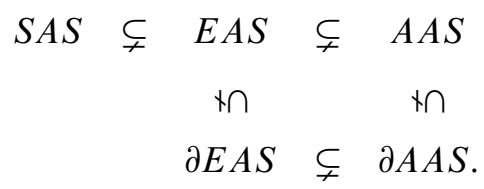

We break the proof of Theorem 4.1 into five propositions below. Each proposition deals with one of the strict containments in the above theorem.

Proposition 4.2. The class of synchronous automaton semigroups is strictly contained in the class of expanding automaton semigroups. 
Proof. Let $m, n \in \mathbb{N}-\{1\}$, and let $S_{m, n}$ denote the semigroup with semigroup presentation $\left\langle a, b \mid b^{m}=b^{n}, a b=b\right\rangle$. We show that $S_{m, n}$ is not a synchronous automaton semigroup for any distinct $m, n$, but $S_{m, n}$ is an expanding automaton semigroup for any distinct $m, n$.

Note that for any distinct $m, n \in \mathbb{N}$ with $m<n$, the rewriting system defined by the rules $a b \rightarrow b$ and $b^{n} \rightarrow b^{m}$ is terminating and confluent. Thus $\left\{b^{j} a^{k} \mid j=\right.$ $1, \ldots, n-1, k \in \mathbb{N}\}$ is a set of normal forms for $S_{m, n}$, and so $a^{r} \neq a^{s}$ in $S_{m, n}$ for any distinct $r, s \in \mathbb{N}$ (i.e., $a$ is not periodic).

We begin by showing $S_{m, n}$ is not a synchronous automaton semigroup. Fix $1<m<n$. Let $\mathcal{A}_{m, n}=(Q, \Sigma, t, o)$ be a synchronous automaton such that $S\left(\mathcal{A}_{m, n}\right)$ is generated by two elements $a$ and $b$ with $b^{m}=b^{n}$ and $a b=b$. We show that $a$ is periodic in $S\left(\mathcal{A}_{m, n}\right)$. Without loss of generality, we assume that $a$ and $b$ are both states of $\mathcal{A}_{m, n}$. Let $\sigma_{1} \in \Sigma$ be such that there exists a minimal number $n>0$ with $a^{n}\left(\sigma_{1}\right)=\sigma_{1}$. Since the action of $a$ is length-preserving, there must exist such a $\sigma_{1}$. Let $\left\{\sigma_{1}, \ldots, \sigma_{n-1}\right\}$ be the orbit of $\sigma_{1}$ under the action of $a$ where $a\left(\sigma_{i}\right)=\sigma_{i+1}$ for $1 \leq i \leq n-2$ and $a\left(\sigma_{n-1}\right)=\sigma_{1}$.

First suppose that $a_{\sigma_{j}}=a^{m_{j}}$ for each $1 \leq j \leq n-1$. If $m_{j}>1$ for some $j$, then $\left(a^{m_{j}}\right)_{\sigma_{j}}=a^{n_{1}}$ where $n_{1}>m_{j},\left(a^{n_{1}}\right)_{\sigma_{j}}=a^{n_{2}}$ where $n_{2}>n_{1}$, and so on. In this case, $a$ will have infinitely many sections, which cannot happen since $a$ is a state of a finite automaton. Thus $m_{j}=1$ for all $j$. Note that if $a^{k}(\sigma)=\sigma_{1}$ for some $k>0$ and $\sigma \in \Sigma$, then the same logic implies that if $a_{\sigma}=a^{r}$ for some $r$ then $r=1$. Thus we see that if $\sigma \in \Sigma$ and the section of $a$ at $a^{k}(\sigma)$ is a power of $a$ for all $k$, then the section of $a$ at $a^{k}(\sigma)$ is $a$ for all $k>0$. Suppose that $a_{\sigma}=a$ for all $\sigma \in \Sigma$. Since the action of $a$ is length-preserving, there exist distinct $r, s \in \mathbb{N}$ such that $\tau_{a}^{r}=\tau_{a}^{s}$. Then, as the only section of $a$ is $a$, we have $a^{r}=a^{s}$.

Suppose now that there is a letter $\sigma \in \Sigma$ such that there exists $\sigma^{\prime}$ in the forward orbit of $\sigma$ under the action of $a$ where $a_{\sigma^{\prime}} \notin\langle a\rangle$. Since $a b=b$ and $b$ is periodic, there exist distinct $m_{\sigma}, n_{\sigma} \in \mathbb{N}$ with $n_{\sigma}>m_{\sigma}$ such that $\left(a^{m_{\sigma}}\right)_{\sigma}=\left(a^{m_{\sigma}+k\left(n_{\sigma}-m_{\sigma}\right)}\right)_{\sigma}$ for any $k \in \mathbb{N}$. To see that this is true, let $t$ be the minimal number such that the orbit of $a^{t}(\sigma)$ under the action of $a$ is a cycle. Since the action of $a$ is length-preserving, there must exist such a $t$. Suppose that there is a $k \in \mathbb{N}$ such that $k \geq t$ and the section of $a$ at $a^{k}(\sigma)$ is $b^{i} a^{j}$ for some $i \in \mathbb{N}$ and $j \in \mathbb{N} \cup\{0\}$. Then the relation $a b=b$ implies that for any $k^{\prime} \geq k$ we have $\left(a^{k^{\prime}}\right)_{\sigma}=b^{i^{\prime}} a^{j}$ for some $i^{\prime}$. Periodicity of $b$ then implies that there are $m_{\sigma}, n_{\sigma} \geq k$ as desired. Suppose, on the other hand, that the section of $a$ at $a^{r}(\sigma)$ is in $\langle a\rangle$ for all $r \geq t$. Let $c$ be the maximal number such that the section of $a$ at $a^{c}(\sigma)$ is not in $\langle a\rangle$ and let $p \in \mathbb{N}$. Then $\left(a^{c+p}\right)_{\sigma}=a^{n_{p}}\left(a^{c}\right)_{\sigma}$ for some $n_{p} \in \mathbb{N}$ and the relation $a b=b$ implies that $\left(a^{c+p}\right)_{\sigma}=\left(a^{c}\right)_{\sigma}$. In this case we let $m_{\sigma}=c$ and $n_{\sigma}=c+1$.

Let $\hat{\Sigma}=\left\{\sigma \in \Sigma \mid\left(a^{r}\right)_{\sigma} \notin\langle a\rangle\right.$ for some $\left.r\right\}$. By the preceding paragraph, for each $\sigma \in \hat{\Sigma}$ choose $m_{\sigma}, n_{\sigma} \in \mathbb{N}$ such that $\left(a^{m_{\sigma}}\right)_{\sigma}=\left(a^{m_{\sigma}+k\left(n_{\sigma}-n_{\sigma}\right)}\right)_{\sigma}$. Since $a$ acts in a length-preserving fashion, there exist distinct $t_{1}, t_{2}$ such that $\tau_{a}^{t_{1}}=\tau_{a}^{t_{1}+k\left(t_{2}-t_{1}\right)}$ for all $k \in \mathbb{N}$. Thus we can choose distinct $s, t \in \mathbb{N}$ such that $\tau_{a^{s}}=\tau_{a^{s+k(t-s)}}$ and 
$\left(a^{s}\right)_{\sigma}=\left(a^{s+k(t-s)}\right)_{\sigma}$ for all $\sigma \in \widehat{\Sigma}$ and $k \in \mathbb{N}$. We claim that $a^{s}=a^{t}$. To see this, let $\delta \in \Sigma$. If $\eta \in \widehat{\Sigma}$, then the choice of $s$ and $t$ implies that $\left(a^{s}\right)_{\eta}=\left(a^{t}\right)_{\eta}$. Fix $\delta \notin \widehat{\Sigma}$. Then $\left(a^{s}\right)_{\delta}=a^{s}$ and $\left(a^{t}\right)_{\delta}=a^{t}$, so the choice of $s$ and $t$ implies that $\tau_{\left(a^{s}\right)_{\delta}}=\tau_{\left(a^{t}\right)_{\delta}}$. If $\eta \in \widehat{\Sigma}$, then

$$
\left(a^{s}\right)_{\delta \eta}=\left(a^{s}\right)_{\eta}=\left(a^{t}\right)_{\eta}=\left(a^{t}\right)_{\delta \eta}
$$

If $\eta \notin \widehat{\Sigma}$ then $\left(a^{s}\right)_{\delta \eta}=a^{s}$ and $\left(a^{t}\right)_{\delta \eta}=a^{t}$, and so $\tau_{\left(a^{s}\right)_{\delta \eta}}=\tau_{\left(a^{t}\right)_{\delta \eta}}$. Similarly, let $w \in \Sigma^{*}$ and write $w=\sigma_{1} \ldots \sigma_{n}$. Suppose there is an $i \in \mathbb{N}$ such that $\sigma_{i} \in \widehat{\Sigma}$ and $\sigma_{1}, \ldots, \sigma_{i-1} \in \Sigma-\widehat{\Sigma}$. Then

$$
\left(a^{s}\right)_{w}=\left(a^{s}\right)_{\sigma_{i} \ldots \sigma_{n}}=\left(a^{t}\right)_{\sigma_{i} \ldots \sigma_{n}}=\left(a^{t}\right)_{w}
$$

On the other hand, if $w \in(\Sigma-\widehat{\Sigma})^{*}$ then $\tau_{\left(a^{s}\right)_{w}}=\tau_{a^{s}}=\tau_{a^{t}}=\tau_{\left(a^{t}\right)_{w}}$. Thus $a^{s}=a^{t}$, and so $S\left(\mathcal{A}_{m, n}\right)$ is not $S_{m, n}$.

Fix $1<m<n$, and let $\Sigma=\left\{\sigma_{1}, \ldots, \sigma_{n}\right\}$ be an alphabet. Let $\mathcal{A}_{m, n}$ be the automaton over the alphabet $\Sigma$ with states $a$ and $b$ (which depend on $m, n$ ) defined by

$$
a=\left[\sigma_{1} \sigma_{1}, \sigma_{2}, \ldots, \sigma_{n}\right](a, \ldots, a), \quad b=\tau_{b}(b, \ldots, b)
$$

where

$$
\tau_{b}\left(\sigma_{i}\right)= \begin{cases}\sigma_{i+1}, & 1 \leq i<n, \\ \sigma_{m}, & i=n .\end{cases}
$$

Then $b^{m}=b^{n}$ in $S\left(\mathcal{A}_{m, n}\right)$. Note also that the range of $b$ is $\left\{\sigma_{2}, \ldots, \sigma_{n}\right\}^{*}$, and $a$ fixes this set. So $a b=b$. Now fix $i, j \in \mathbb{N}$ such that $i<n$. Then $b^{i} a^{j}\left(\sigma_{1}\right)=$ $b^{i}\left(\sigma_{1}^{2^{j}}\right)=\sigma_{i}^{2^{j}}$. Thus $b^{i} a^{j}=b^{k} a^{l}$ in $S\left(\mathcal{A}_{m, n}\right)$ if and only if $i=k$ and $j=l$, and we have $S\left(\mathcal{A}_{m, n}\right) \cong S_{m, n}$.

Recall that the bicyclic monoid is the monoid with presentation $B:=\operatorname{Mon}\langle a, b|$ $a b=1\rangle$. This monoid is not residually finite (see chapter 5 of [9] by Lallement), and in Proposition 5.1 below we show that expanding automaton semigroups are residually finite. Thus $B$ is not a submonoid of any expanding automaton semigroup. We use this fact to distinguish the class of expanding automaton semigroups from the class of asynchronous automaton semigroups.

Proposition 4.3. The class of expanding automaton semigroups is strictly contained in the class of asynchronous automaton semigroups.

Proof. We show the proposition by demonstrating an asynchronous automaton that gives the bicyclic monoid.

Consider the asynchronous automaton given in Figure 4. We show that $S(\mathcal{A})$ has presentation $\operatorname{Mon}\langle a, b \mid b a=1\rangle$, and so $S(\mathcal{A})$ is the bicyclic monoid.

Note that $e$ pointwise fixes $\{0,1\}^{*}$, and hence $e$ is an identity element of $S(\mathcal{A})$. Let $w \in\{0,1\}^{*}$. Then $b a(0 w)=b(00 w)=0 w$ and $b a(1 w)=b(11 w)=1 w$. Hence 


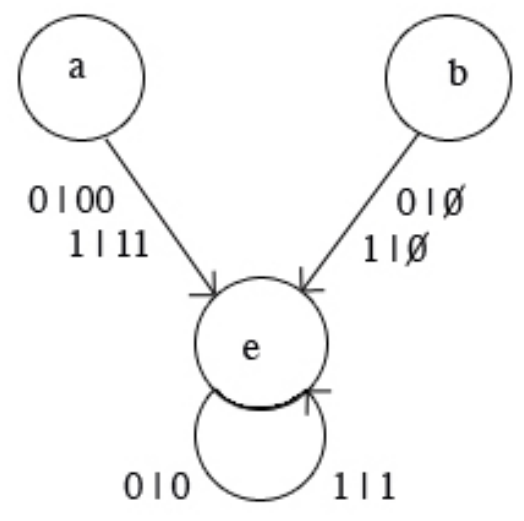

Figure 4. The automaton from Proposition 4.3.

$b a=e$. On the other hand, $a b(0)=a(\emptyset)=\emptyset$, and so $a b \neq e$. Corollary 1.32 of the book [2] by Clifford and Preston then implies that $S(\mathcal{A})$ is the bicyclic monoid.

Let $\mathbb{N}^{0}$ denote the free semigroup of rank 1 with a zero adjoined, i.e., $\mathbb{N}^{0}=$ $S g\left\langle a, b \mid b^{2}=b, a b=b a=b\right\rangle$. We show below in Proposition 4.5 that $\mathbb{N}^{0}$ is not an expanding automaton semigroup, but that $\mathbb{N}^{0}$ is a $\partial$-expanding automaton semigroup. First, we need the following lemma.

Lemma 4.4. $\mathbb{N}^{0}$ is not a synchronous automaton semigroup.

Proof. Suppose $S$ is a synchronous automaton semigroup over an alphabet $\Sigma=$ $\left\{\sigma_{1}, \ldots, \sigma_{n}\right\}$ such that $S$ is generated by two elements $a$ and $b$ with $a b=b a=b$ and $b^{2}=b$. We use the same idea of the proof of Proposition 4.2 to show that $a$ is periodic.

Let $\sigma \in \Sigma$. Suppose that the section of $a$ at $a^{n}(\sigma)=b$ for some $n$. Then $\left(a^{n}\right)_{\sigma}=\left(a^{n+k}\right)_{\sigma}$ for all $k \in \mathbb{N}$. If the section of $a$ at $a^{n}(\sigma)$ is a power of $a$ for all $n$, then (as in the proof of Proposition 4.2) the section of $a$ at $a^{n}(\sigma)$ is $a$ for all $n$.

Let $\widehat{\Sigma}=\left\{\sigma \in \Sigma \mid\left(a^{r}\right)_{\sigma}=b\right.$ for some $\left.r\right\}$. As in the proof of Proposition 4.2, we can choose $s$ and $t$ such that $\tau_{a^{s}}=\tau_{a^{t}}$ and $\left(a^{s}\right)_{\sigma}=\left(a^{t}\right)_{\sigma}$ for all $\sigma \in \hat{\Sigma}$. Then the logic of the proof of Proposition 4.2 shows that $a^{s}=a^{t}$.

Proposition 4.5. The class of expanding automaton semigroups is strictly contained in the class of $\partial$-expanding automaton semigroups.

Proof. First we show containment. Let $\mathcal{A}=(Q, \Sigma, t, o)$ be an expanding automaton. Let $x$ be a symbol not in $\Sigma$, and let $\Sigma^{\prime}=\Sigma \cup\{x\}$. We construct a new automaton $\mathscr{B}=\left(Q, \Sigma^{\prime}, t^{\prime}, o^{\prime}\right)$ such that $S(\mathscr{B}) \cong S(\mathcal{A})$ and $\partial S(\mathscr{B}) \cong S(\mathscr{B})$. If $(q, \sigma) \in Q \times \Sigma$, define $t^{\prime}(q, \sigma)=t(q, \sigma)$. For each $q \in Q$, let $t^{\prime}(q, x)=q$. Similarly, for each 
$(q, \sigma) \in Q \times \Sigma$ let $o^{\prime}(q, \sigma)=o(q, \sigma)$ and $o^{\prime}(q, x)=x$. Then $S(\mathscr{B}) \cong S(\mathcal{A})$. Let $q_{1}, q_{2} \in Q^{*}$ be distinct elements of $S(\mathcal{A})$. Then there is a $w \in \Sigma^{*}$ such that $q_{1}(w) \neq q_{2}(w)$. Now $q_{1}\left(w x^{\omega}\right)=q_{1}(w) x^{\omega} \neq q_{2}(w) x^{\omega}=q_{2}\left(w x^{\omega}\right)$, and so $q_{1}$ and $q_{2}$ are distinct elements of $\partial S(\mathscr{B})$. Since $\partial S(\mathscr{B})$ is a quotient of $S(\mathscr{B})$, we have shown that $S(\mathscr{B}) \cong \partial S(\mathscr{B})$.

We now show that $\mathbb{N}^{0}$ is not an expanding automaton semigroup. Let $S=$ $\left\langle a, b \mid b^{2}=b, a b=b a=b\right\rangle$ be the free semigroup of rank 1 with a zero adjoined, and suppose $S$ were an expanding automaton semigroup corresponding to the automaton $\mathcal{A}=(Q, \Sigma, t, o)$. Since $b$ is idempotent, $b$ fixes range $(b)$. Hence the set $\hat{\Sigma}=\{\sigma \in \Sigma \mid b(\sigma)=\sigma\}$ is non-empty. Since $b$ is the only idempotent of $S$, $b_{\hat{\sigma}}=b$ for all $\hat{\sigma} \in \hat{\Sigma}$.

Let $\sigma \in \Sigma-\hat{\Sigma}$, and suppose that $b_{\sigma}=a^{n}$ for some $n>0$. Let $w \in \Sigma^{*}$. Then $b(\sigma w)=b(\sigma) a^{n}(w)$. Since $b$ fixes range $(b)$, we have that $b(b(\sigma w))=b(\sigma) a^{n}(w)$. We also have that $b$ fixes $b(\sigma)$ and the section of $b$ at $b(\sigma)$ is $b$. Thus $b$ fixes $a^{n}(w)$, and (as $w$ is arbitrary) $b a^{n}=a^{n}$ in $S$. But $b a^{n}=b$, which implies that $a^{n}$ is idempotent. Since $a^{n}$ is not idempotent in $S$, we have $b_{\sigma}=b$ for all $\sigma \in \Sigma$. Note that $b$ must be a state of $\mathcal{A}$ as powers of $a$ cannot multiply to obtain $b$. Thus, in the graphical representation of $\mathcal{A}$, all edges going out of $b$ are loops based at $b$. Note also that $a$ must be a state of $\mathcal{A}$.

Let $\Gamma=\left\{\sigma \in \Sigma:\left|a^{m}(\sigma)\right|=1\right.$ for all $\left.m\right\}$. The equation $a b=b$ implies that $a$ fixes range $(b)$, and so $\Gamma$ is nonempty. In $\mathcal{A}$, for each state $q$ in $\langle a\rangle$ and $\gamma \in \Gamma$ there is an arrow labeled by $\gamma \mid \hat{\gamma}$ coming out of $q$ where $\hat{\gamma} \in \Gamma$. Let $w \in \Gamma^{*}$ with $w=\gamma_{1} \ldots \gamma_{k}$. Suppose that $|a(w)|>1$. Then $w$, as a path in $\mathcal{A}$ based at $a$, must enter the state $b$. Choose $i$ maximal so that $\gamma_{1} \ldots \gamma_{i-1}$ is a path such that the initial vertex of each edge is not the state $b$. Then $a(w)=\gamma_{1}^{\prime} \ldots \gamma_{k}^{\prime}$ where $\gamma_{m}^{\prime} \in \Gamma$ for $1 \leq m \leq i-1$ and $\gamma_{m}^{\prime} \in \hat{\Sigma}^{*}$ for $i \leq m \leq k$. Since $a$ fixes $\hat{\Sigma}^{*},\left|a^{n}(w)\right|=\left|a^{2}(w)\right|$ for all $n \geq 2$. Thus for any $w \in \Gamma^{*},\left|a^{|\Sigma|}(w)\right|=\left|a^{k}(w)\right|$ for any $k \geq|\Sigma|$.

Suppose that $|a(\sigma)|=1$ for all $\sigma \in \Sigma$. Then the same logic as in the proof of Proposition 4.2 shows that either $a$ is periodic or has infinitely many sections (note that the proof does not use that the periodic element acts in a length-preserving fashion). So the set $\Sigma^{\prime}=\{\sigma \in \Sigma:|a(\sigma)|>1\}$ is nonempty. Let $\sigma^{\prime} \in \Sigma^{\prime}$, and write $a\left(\sigma^{\prime}\right)=\sigma_{1} \ldots \sigma_{m}$ where $\sigma_{i} \in \Sigma$. Suppose that $\sigma_{i}=\sigma^{\prime}$ for some $i$. Then $b\left(a\left(\sigma^{\prime}\right)\right)=b\left(\sigma_{1} \ldots \sigma_{n}\right)=b\left(\sigma_{1}\right) \ldots b\left(\sigma_{m}\right)=b\left(\sigma^{\prime}\right)$, and so $\left|b\left(a\left(\sigma^{\prime}\right)\right)\right|>\left|b\left(\sigma^{\prime}\right)\right|$, a contradiction. Thus $\sigma^{\prime}$ is not a letter of $a\left(\sigma^{\prime}\right)$. The same calculation also shows that $\sigma^{\prime}$ is not a letter of $a^{n}\left(\sigma^{\prime}\right)$ for any $n$ and that $\sigma^{\prime}$ is not a letter of $a\left(\sigma_{i}\right)$ for any $i$.

Let $w \in \Sigma^{*}$ and write $w=\sigma_{1} \ldots \sigma_{k}$. Suppose that $\sigma_{i} \notin \Gamma$ for some $i$. Then every edge in $\mathcal{A}$ with input label $\sigma_{i}$ has an output label without $\sigma_{i}$ as a letter. Thus $a^{n}(w)$ does not contain $\sigma_{i}$ as a letter for any $n$. If $a(w) \in \Gamma^{*}$, then as mentioned above $a$ will act in a length-preserving fashion on $a^{|\Sigma|}(w)$. Suppose that $a(w) \notin \Gamma^{*}$ where $\sigma_{j} \notin \Gamma$ is a letter of $a(w)$. Then $a^{2}(w)$ does not contain $\sigma_{i}$ or $\sigma_{j}$ as a letter. Continuing inductively, we see that $a^{|\Sigma|}(w) \in \Gamma^{*}$. Thus there is an $m \in \mathbb{N}$ such that $a$ acts in a length-preserving fashion on $a^{m}(w)$ for any $w \in \Sigma^{*}$, i.e., $\left|a^{m}(w)\right|=\left|a^{k}(w)\right|$ 
for $k \geq m$ and any $w \in \Sigma^{*}$. This induces a length-preserving action of $S$ on $\Gamma^{*}$, contradicting Lemma 4.4. Hence $\mathbb{N}^{0}$ is not an expanding automaton semigroup.

To see that $\mathbb{N}^{0}$ is a $\partial$-expanding automaton semigroup, consider the automaton given by the wreath decomposition $a=[01,11](b, a), b=[01,01](b, b)$. Then the range of $b^{\omega}$ is $\eta:=010101 \cdots$. By construction of the automaton, $a^{\omega}$ fixes $\eta$ and so $a b=b$. Since the range of $b^{\omega}$ is a single point, $b^{\omega} a^{\omega}=b^{\omega}$. Finally, note that $a^{\omega}$ is not periodic as $\left(a^{\omega}\right)^{n}(1 \eta)=1^{2^{n}} \eta$, i.e., $1 \eta$ has an infinite forward orbit under the action of $a^{\omega}$. Thus the semigroup generated by $a^{\omega}$ and $b^{\omega}$ is $\mathbb{N}^{0}$.

Proposition 4.6. The class of d-expanding automaton semigroups is strictly contained in the class of $\partial$-asynchronous automaton semigroups.

Proof. Containment follows from the definitions of the kinds of automata. We show in Proposition 5.2 that the bicyclic monoid is not a submonoid of any $\partial$-expanding automaton semigroup, thus to show strict containment we show that the bicyclic monoid is an a $\partial$-asynchronous automaton semigroup.

Let $\mathcal{A}$ be the automaton from Figure 4 . Then $e^{\omega}$ is an identity element of $\partial S(\mathcal{A})$. Let $\eta \in \Sigma^{\omega}$. Then $b^{\omega} a^{\omega}(0 \eta)=b^{\omega}(00 \eta)=0 \eta$ and $b^{\omega} a^{\omega}(1 \eta)=b^{\omega}(11 \eta)=1 \eta$. Hence $b^{\omega} a^{\omega}=e^{\omega}$. On the other hand, $a^{\omega} b^{\omega}(0111 \cdots)=a^{\omega}(111 \cdots)=111 \cdots$, and so $a^{\omega} b^{\omega} \neq e^{\omega}$. Thus Corollary 1.32 of [2] implies that $\partial S(\mathcal{A})$ is the bicyclic monoid.

Proposition 4.7. The class of asynchronous automaton semigroups is strictly contained in the class of boundary asynchronous automaton semigroups.

Proof. To show containment, the logic and constructions are the same as those at the beginning of the proof of Proposition 4.5.

To see that the containment is strict, note that Grigorchuk et al. in [4] demonstrate a $\partial$-asynchronous automaton semigroup $S$ such that Thompson's group $F$ is a subgroup of $S$ (see section 5.2 of [4]). Recall that $F$ is an infinite simple group, and so $F$ is not residually finite. Thus $S$ is not an asynchronous automaton semigroup, because we show in Proposition 5.7 below that subgroups of asynchronous semigroups are residually finite.

At this time, we are unsure of the relationship between the class of $\partial$-expanding automaton semigroups and the class of asynchronous automaton semigroups. We can say that the class of asynchronous automaton semigroups is not contained in the class of $\partial$-expanding automaton semigroups because the bicyclic monoid only appears in one class, but we are unsure whether or not something stronger is true.

\section{Algebraic theory}

We now turn to studying the algebraic properties of semigroups given by asynchronous automata. 


\subsection{Residual finiteness and the absence of the bicyclic monoid}

Proposition 5.1. Expanding automaton semigroups are residually finite.

Proof. Let $S$ be an expanding automaton semigroup over the alphabet $\Sigma$ and let $a, b \in S$ with $a \neq b$. For each $m \in \mathbb{N}$, let $L(m)=\left\{w \in \Sigma^{*}:|w|=m\right\}$, i.e., $L(m)$ is the $m$ th level of the tree $\Sigma^{*}$. Since $a$ and $b$ are distinct, there is $n \in \mathbb{N}$ such that $a$ and $b$ act differently on $L(n)$. Let

$$
n^{\prime}=\max \{|a(w)|,|b(w)|: w \in L(n)\}
$$

and let $\mathscr{L}=\left(\bigcup_{i=1}^{n^{\prime}} L(i)\right) \cup\{\$\}$. Finally, let $T(\mathscr{L})$ denote the semigroup of transformations $\mathscr{L} \rightarrow \mathscr{L}$. Since $\mathscr{L}$ is finite, $T(\mathscr{L})$ is a finite semigroup. Define a homomorphism $\rho: S \rightarrow T(\mathscr{L})$ by $\rho(s)=f$ where $f(\$)=\$$ and

$$
f(x)= \begin{cases}s(x), & s(x) \in \mathscr{L}, \\ \$, & s(x) \notin \mathscr{L} .\end{cases}
$$

Since $a$ and $b$ act differently on $L(n)$, construction of $\rho$ ensures that $\rho(a)$ and $\rho(b)$ are distinct in $T(\mathscr{L})$.

Proposition 5.2. Let $S$ be a d-expanding automaton semigroup. Then the bicyclic monoid is not a submonoid of $S$.

Proof. Let $\mathcal{A}=(Q, \Sigma, t, o)$ be an expanding automaton and let $S=\partial S(\mathcal{A})$. Suppose that there exists elements $a, b, c \in S$ such that $c^{2}=c, a c=c a=a, b c=$ $b c=b$, and $b a=c$. We show that $a b=c$, and hence the subsemigroup generated by $a, b$, and $c$ is not the bicyclic monoid.

Since $c a=a$ and $c b=b, c$ fixes range $(a)$ and range $(b)$. Thus range $(a) \subseteq$ range $(c)$. Since $b a=c, b(\operatorname{range}(a))=\operatorname{range}(c)$. Because $c$ is a continuous transformation of $\Sigma^{\omega}$, range $(c)$ is a compact subset of $\Sigma^{\omega}$. Since $\mathcal{A}$ is an expanding automaton, $b$ is either an isometry or a contraction mapping of range $(c)$. Thus if $\operatorname{range}(a) \subsetneq \operatorname{range}(c)$, then $b(\operatorname{range}(a)) \subsetneq \operatorname{range}(c)$. Hence range $(a)=\operatorname{range}(c)$. Thus both $a$ and $b$ are isometries on range $(c)$, and therefore $a b=c$ on range $(c)$. Let $\eta \notin$ range $(c)$. Then $b a b(\eta)=b(\eta)=b c(\eta)$. Since $a b(\eta), c(w) \in \operatorname{range}(c)$ and $b$ acts injectively on range $(c)$, we have $a b(\eta)=c(\eta)$. Thus $a b=c$.

Since the bicyclic monoid is not a submonoid of any $\partial$-expanding automaton semigroup, an element $s$ of $\partial$-expanding automaton semigroup is left invertible if and only if $s$ is right-invertible.

Remark 5.3. Let $S$ be a $\partial$-expanding automaton semigroup and let $M$ be a submonoid of $S$ with identity 1 . If $a, b \in M$ and $a b=1$, then $b a=1$. In other words, an element $s$ of a monoid arising from an expanding automaton is left invertible if and only if $s$ is right invertible. 
5.2. Groups. In this section we investigate the subgroup structure of $\partial$-expanding automaton semigroups and asynchronous automaton semigroups. First, some notation: let $\mathcal{A}=(Q, \Sigma, t, o)$ be an invertible synchronous automaton. Then the inverse automaton for $\mathcal{A}$, denoted by $\mathcal{A}^{-1}$, is the automaton with state set $Q^{-1}$, alphabet $\Sigma$, transition function $t^{-1}$, and output function $o^{-1}$. The transition and output functions are defined by $t^{-1}\left(q_{1}^{-1}, \sigma\right)=q_{2}^{-1}$ if and only if $t\left(q_{1}, \sigma\right)=q_{2}$ and $o^{-1}\left(q^{-1}, \sigma_{1}\right)=\sigma_{2}$ if and only if $o\left(q, \sigma_{2}\right)=\sigma_{1}$. This automaton is called the inverse automaton because $q q^{-1}$ is the automorphism that pointwise fixes $\Sigma^{*}$. Let $\mathcal{A}^{ \pm 1}$ denote $\mathcal{A} \cup \mathcal{A}^{-1}$.

Proposition 5.4. A group $G$ is a $\partial$-expanding automaton semigroup (respectively $\partial$ expanding self-similar semigroup) if and only if $G$ is an automaton group (respectively self-similar group).

Before we begin the proof, recall that an inactive path in an automaton $\mathcal{A}=$ $(Q, \Sigma, t, o)$ is a (possibly empty) path in $\mathcal{A}$ such that every edge on the path has a label of the form $\sigma \mid \sigma$.

Proof. Let $G$ be an automaton group corresponding to the invertible synchronous automaton $\mathcal{A}:=(Q, \Sigma, t, o)$. Construct a new automaton $\mathscr{B}=\mathcal{A} \cup \mathcal{A}^{-1}$. Then $\partial S(\mathscr{B})=G$ and $\mathscr{B}$ is an expanding automaton. Thus $G$ is an expanding automaton semigroup.

Conversely, let $\mathcal{A}$ be an expanding automaton such that $\partial S(\mathcal{A}):=G$ is a group. Let $e$ be a state of $\mathcal{A}$ such that $e^{\omega}$ is the identity element of $G$. Since $e^{\omega}$ is idempotent, $e^{\omega}$ fixes its range in $\Sigma^{\omega}$. In order for $e^{\omega}$ to have a fixed point in $\Sigma^{\omega}$, there must exist either (a) an inactive circuit in $\mathcal{A}$ accessible from $e$ via an inactive path or (b) an edge in $\mathcal{A}$ with a label of the form $\sigma \mid \sigma w$ for some $w \in \Sigma^{+}$accessible from $e$ via an inactive path (see Remark 3.5 above).

Let $q \in Q$. Since $q^{\omega} \in G$, range $\left(q^{\omega}\right)=\operatorname{range}\left(e^{\omega}\right)$ and $q^{\omega}$ acts bijectively on range $\left(e^{\omega}\right)$.

Since $e^{\omega}$ is the only idempotent of $S$, any inactive path in $\mathcal{A}$ that begins at $e$ must also end at $e$. So any fixed point of $e^{\omega}$ that arises from condition (a) above must define a path in $\mathcal{A}$ that never leaves $e$.

Suppose there is an edge $\gamma$ in $\mathcal{A}$ such that $\gamma$ begins at $e$ and $\gamma$ has a label of the form $\sigma \mid \sigma w$ for some $w \in \Sigma^{+}$, i.e., $e^{\omega}$ has a fixed point arising from condition (b) above. Recall from the proof of Proposition 3.4 that, in this case, $e^{\omega}$ has precisely one fixed point in $\sigma \Sigma^{\omega}$. Furthermore, all words in $\sigma \Sigma^{\omega}$ must be mapped to this fixed point by $e^{\omega}$ because $e^{\omega}$ is idempotent. Hence the range of $\left(e_{\sigma}\right)^{\omega}$ is a single point, and hence $\left(e_{\sigma}\right)^{\omega}$ is idempotent. Thus $\gamma$ must be a circuit at $e$. Suppose there is another circuit at $e$ with a label of the form $\delta \mid \delta$ where $\delta \in \Sigma$. Now $e^{\omega}$ has a unique fixed point $x$ in $\sigma \Sigma^{\omega}$, and all other words in $\sigma \Sigma^{\omega}$ must be mapped to $x$ by $e^{\omega}$. But $e^{\omega}(\sigma \delta \delta \delta \cdots)=\sigma w \delta \delta \delta \cdots$ and $e^{\omega}(\sigma \sigma \delta \delta \delta \cdots)=\sigma w \sigma w \delta \delta \delta \cdots$. Thus if $e^{\omega}$ has a fixed point arising from condition (b) above, then $e^{\omega}$ does not have a fixed point arising from condition (a) above. Hence, in the case that $e^{\omega}$ has a fixed point arising 
from condition (b), we have that $e^{\omega}$ has only finitely many fixed points. This implies that $G$ is a finite group, and any finite group is self-similar.

Now suppose that $e^{\omega}$ has a fixed point in $\Sigma^{\omega}$ arising from condition (a) above. Then there is a subalphabet $\widehat{\Sigma}$ such that for all $\hat{\sigma} \in \hat{\Sigma}$ there is a circuit of the form $\hat{\sigma} \mid \hat{\sigma}$ at $e$. In addition, the range of $e^{\omega}$ is $\hat{\Sigma}^{\omega}$ and hence $G$ acts bijectively on $\hat{\Sigma}^{\omega}$. Let $q \in Q$ and suppose that there is an edge coming from $q$ of the form $\hat{\sigma} \mid w$ where $\hat{\sigma}, w \in \widehat{\Sigma}^{*}$ and $w$ has length greater than 1 . Then $q^{\omega}$ is a contracting map on $\hat{\Sigma}^{\omega}$. Since $\mathcal{A}$ is an expanding automaton, every element of $G$ is either a contraction or an isometry of $\widehat{\Sigma}^{\omega}$. Since $\hat{\Sigma}^{\omega}$ is compact, this would imply that $q^{\omega} \notin G$. Hence if there is an edge in the automaton of the form $\hat{\sigma} \mid w$ where $\hat{\sigma}, w \in \hat{\Sigma}$, then $w$ has length 1 . Thus $G \cong \partial S(\mathscr{B})$, where $\mathscr{B}$ is the invertible synchronous subautomaton of $\mathcal{A}$ corresponding to $\hat{\Sigma}$, and hence $G$ is an automaton group.

None of the above work used the finiteness of the automaton, and hence the corresponding proposition for self-similarity is also true.

We can use the ideas of the previous proof to show the following proposition.

Proposition 5.5. Let $S$ be a d-expanding automaton semigroup with a unique maximal subgroup $G$. Then $G$ is self-similar.

Proof. Let $\mathcal{A}=(Q, \Sigma, t, o)$ be an expanding automaton such that $\partial S(\mathcal{A})$ has a unique maximal subgroup $G$. Let $e$ be a state of $\mathcal{A}$ such that $e^{\omega}$ is the unique idempotent of $\partial S(\mathcal{A})$. As in the proof of Proposition 5.4, if there is an edge coming out of $e$ with a label of the form $\sigma \mid \sigma w$ with $w \in \Sigma+$, then $e^{\omega}$ has finitely many fixed points and hence $G$ is a finite group. Hence we assume that there is a subalphabet $\widehat{\Sigma}$ of $\Sigma$ such that $e(\hat{\sigma})=\hat{\sigma}$ and $e_{\hat{\sigma}}=e$ for all $\hat{\sigma} \in \hat{\Sigma}$, and thus if $\eta \in \Sigma^{\omega}$ is a fixed point of $e^{\omega}$ then $\eta \in \hat{\Sigma}^{\omega}$.

Let $\sigma \in \hat{\Sigma}$ and let $g \in G$. We assume without loss of generality that there is a state $q \in Q$ such that $q^{\omega}=g$. Write $e=\tau_{e}\left(e_{1}, \ldots, e_{n}\right)$ and $q=\tau_{q}\left(q_{1}, \ldots, q_{n}\right)$. Then $\tau_{q}(\sigma) \in \widehat{\Sigma}$ and so $e_{\tau_{q}(\sigma)}=e$. Thus Equation (2) implies

$$
q_{\sigma}=(e q)_{\sigma}=e_{\tau_{q}(\sigma)} q_{\sigma}
$$

and, as $e$ stabilizes $\sigma$,

$$
q_{\sigma}=(q e)_{\sigma}=q_{\sigma} e_{\sigma} .
$$

Hence $e^{\omega}\left(q_{\sigma}\right)^{\omega}=\left(q_{\sigma}\right)^{\omega} e^{\omega}=\left(q_{\sigma}\right)^{\omega}$ for any $\sigma \in \hat{\Sigma}$.

Let $h=g^{-1}$, and assume without loss of generality that there is a state $r \in Q$ such that $r_{\omega}=h$. Write $r=\left(r_{1}, \ldots, r_{n}\right) \tau_{r}$ and let $\sigma \in \hat{\Sigma}$. By the same logic as above, $e^{\omega}\left(r_{\sigma}\right)^{\omega}=\left(r_{\sigma}\right)^{\omega} e^{\omega}=\left(r_{\sigma}\right)^{\omega}$. Since $h g=e$ we have

$$
\left((r q)_{\sigma}\right)^{\omega}=\left(r_{\tau_{q}(\sigma)} q_{\sigma}\right)^{\omega}=\left(e_{\sigma}\right)^{\omega}=e^{\omega} .
$$

Since $\left(q_{\sigma}\right)^{\omega}$ is left-invertible, Remark 5.3 implies that $\left(q_{\sigma}\right)^{\omega}$ is invertible. Therefore $\left(q_{\sigma}\right)^{\omega} \in G$ for all $\sigma \in \widehat{\Sigma}$. 
Continuing inductively, we see that $\left(q_{v}\right)^{\omega} \in G$ for all $v \in \operatorname{range}(e)$. Similar to the proof of Proposition 5.4, we can consider the action of $G$ only on $\hat{\Sigma}^{\omega}$. Thus $G$ is an expanding self-similar semigroup, and Proposition 5.4 implies that $G$ is a self-similar group.

If $\mathcal{A}$ is an invertible synchronous automaton, then $S(\mathcal{A})$ has at most one idempotent, namely the idempotent that pointwise fixes the corresponding tree. Hence Proposition 5.5 has the following corollary.

Corollary 5.6. Let $\mathcal{A}$ be an invertible synchronous automaton. Then the group of units of $S(\mathcal{A})$ is self-similar.

We conclude this section by showing that subgroups of asynchronous automaton semigroups are residually finite.

Proposition 5.7. If $G$ is a subgroup of an asynchronous self-similar semigroup, then $G$ is residually finite.

Proof. Let $\mathcal{A}=(Q, \Sigma, t, o)$ be an asynchronous automaton where $|Q|$ can be infinite, let $S=S(\mathcal{A})$, and let $G$ be a subgroup of $S$. Let $e$ denote the identity element of $G$. Since $e$ is idempotent, $e$ fixes range $(e)$. Since $G$ is a group, $G$ acts bijectively on range $(e)$.

If $v, w \in \Sigma^{*}$, write $v<w$ if $v$ is a proper prefix of $w$. Let $L(n)$ denote the set of elements $v \in \operatorname{range}(e)$ such that there exists a chain $\emptyset<v_{1}<\cdots<v_{n-1}<v$ of maximal length where $v_{i} \in \operatorname{range}(e)$ for all $1 \leq i \leq n-1$.

Let $g \in G$. Then $g(\emptyset)=\emptyset$. Since $g$ acts bijectively on range $(e), g$ cannot send any point of range $(e)$ to $\emptyset$. Let $v \in L(1)$. Then there is a point $w \in \operatorname{range}(e)$ such that $g(w)=v$. If $w \notin L(1)$, then let $w_{1} \in L(1)$ be proper prefix of $w$. Since $g(w)=v$ and $g$ acts in a prefix-preserving fashion, $g\left(w_{1}\right)=\emptyset$ or $g\left(w_{1}\right)=v$. In either case, $g$ does not act bijectively on range $(e)$. Thus $w \in L(1)$. Since $L(1)$ is finite, $g$ induces a permutation of $L(1)$. Inductively, $g$ induces a permutation of $L(n)$ for all $n$.

Let $\operatorname{Stab}(L(n))=\{g \in G \mid g(v)=v$ for all $v \in L(n)\}$. The previous paragraph implies that $\operatorname{Stab}(L(n))$ is a finite index, normal subgroup of $G$. If $h$ is a non-trivial element of $G$, then there must exist a word $v \in L(m)$ for some $m$ such that $h(v) \neq v$. Thus $\operatorname{Stab}(L(m))$ is a finite index, normal subgroup that does not contain $h$, and so $G$ is residually finite.

Acknowledgements. Thank you to Susan Hermiller and John Meakin for their advice and support. 


\section{References}

[1] A. J. Cain, Automaton semigroups. Theoret. Comput. Sci. 410 (2009), 5022-5038. Zbl 1194.68133 MR 2583696

[2] A. H. Clifford and G. B. Preston, The algebraic theory of semigroups. Vol. I . Math. Surveys 7, Amer. Math. Soc., Providence, R.I., 1961. Zbl 0111.03403 MR 0132791

[3] R. I. Grigorchuk, On the Milnor problem of group growth. Dokl. Akad. Nauk SSSR 271 (1983), 30-33; English transl. Soviet Math. Dokl. 28 (1983), 23-26. Zbl 0547.20025 MR 712546

[4] R. I. Grigorchuk, V. V. Nekrashevich, and V. I. Sushchanskii, Automata, dynamical systems, and groups. Trudy Mat. Inst. Steklova 231 (2000), 134-214; English transl. Proc. Steklov Inst. Math. 231 (2000), 128-203. Zbl 1155.37311 MR 1841755

[5] R. Grigorchuk and Z. Šunić, Self-similarity and branching in group theory. In Groups St. Andrews 2005, Vol. 1, London Math. Soc. Lecture Note Ser. 339, Cambridge Univ. Press, Cambridge 2007, 36-95. Zbl 1185.20044 MR 2327317

[6] R. I. Grigorchuk and A. Żuk, The lamplighter group as a group generated by a 2-state automaton, and its spectrum. Geom. Dedicata 87 (2001), 209-244. Zbl 0990.60049 MR 1866850

[7] J. E. Hopcroft, J. D. Ullman, and R. Motwani, Introduction to automata theory, languages, and computation. 2nd ed., Addison-Wesley Publishing Co., Reading, MA, 2001. Zbl 0980.68066 MR 645539

[8] G. Lallement, Semigroups and combinatorial applications. John Wiley \& Sons, New York-Chichester-Brisbane 1979. Zbl 0421.20025 MR 530552

[9] M. Lothaire, Combinatorics on words. Encyclopedia Math. Appl. 17, Cambridge University Press, Cambridge 1997. Zbl 0874.20040 MR 1475463

[10] D. McCune, Groups and semigroups generated by automata. Ph.D. thesis, University of Nebraska-Lincoln, 2011.

[11] V. Nekrashevych, Self-similar groups. Math. Surveys Monogr. 117, Amer. Math. Soc., Providence, RI, 2005. Zbl 1087.20032 MR 2162164

[12] I. I. Reznikov and V. I. Sushchanskii, Growth functions of two-state automata over a twoelement alphabet. Dopov. Nats. Akad. Nauk Ukr. Mat. Prirodozn. Tekh. Nauki (2002), 76-81. MR 1916771

[13] K. Ruohonen, Reversible machines and Post's correspondence problem for biprefix morphisms. Elektron. Informationsverarb. Kybernet. 21 (1985), 579-595. Zbl 0604.68057 MR 825861

[14] P. V. Silva and B. Steinberg, On a class of automata groups generalizing lamplighter groups. Internat. J. Algebra Comput. 15 (2005), 1213-1234. Zbl 1106.20028 MR 2197829

[15] J. K. Slupik and V. I. Sushchansky, Inverse semigroups generated by two-state partially defined automata. In Proceedings of the 68th workshop on general algebra "68. Arbeitstagung Allgemeine Algebra”, Heyn, Klagenfurt 2005, 261-273. Zbl 1079.20080 MR 2166964 
Received November 3, 2011; revised August 8, 2012

D. McCune, William Jewell College, Department of Mathematics and Physics, 500 William Jewell College Dr, Liberty, MO 64068, U.S.A.

E-mail: mccuned@william.jewell.edu 